\title{
Caracterização da assembléia fitobentônica da praia do Kutuca, ilha da Marambaia, baía de Sepetiba, RJ, Brasil
}

\author{
Renata Perpetuo Reis ${ }^{1,2}$
}

Recebido em 16/10/2007. Aceito em 26/05/2008

RESUMO - (Caracterização da assembléia fitobentônica da praia do Kutuca, ilha da Marambaia, baía de Sepetiba, RJ, Brasil). Devido aos potenciais problemas ambientais na baía de Sepetiba, sítios de monitoramento ambiental nesta baía se tornam necessários. A praia do Kutuca foi selecionada para este propósito por possuir dados pretéritos sobre a sua estrutura da comunidade, com coletas realizadas em 1999, que detectou alta diversidade. Durante 21 meses, dezembro de 2003 a julho de 2005, foram coletados 63 táxons (Chlorophyta, $22 \%$; Ochrophyta, $16 \%$ e Rhodophyta, $62 \%)$. Na amostragem destrutiva, foram usados seis quadrados aleatórios $(25 \times 25 \mathrm{~cm})$, colocados em cada uma das duas linhas de $20 \mathrm{~m}$ horizontais ao costão. Os resultados de 1999 foram comparados com estes e se observou que a biomassa passou de 490,9 $\pm 201,2 \mathrm{~g} \cdot \mathrm{m}^{-2}$ para $199,57 \pm 29,33 \mathrm{~g} \cdot \mathrm{m}^{-2}$, a riqueza de $13,0 \pm 4,5$ para $5,06 \pm 1,72$, a diversidade $\mathrm{H}^{\prime}=2,2 \pm 0,41$ para $\mathrm{H}^{\prime}=1,3 \pm 0,39$ e a equitabilidade $\mathrm{J}^{\prime}=0,65 \pm 0,06$ para $\mathrm{J}^{\prime}=0,55 \pm 0,17$.Quatro táxons (Sargassum spp., Caulerpa sertularioides (S.G. Gmel.) M. Howe, Hypnea musciformis (Wulfen in Jacquin) J.V. Lamour. e Gracilaria cervicornis (Turner) J. Agardh) mais representativos contribuíram com 15 a 33\% de biomassa, enquanto que em 1999, oito táxons (Caulerpa sertularioides, Dictyopteris delicatula J.V. Lamour., Gracilaria cervicornis, Sargassum spp., Codium taylorii P.C. Silva, Padina gymnospora (Kützing) Sonder, Galaxaura marginata (Ellis \& Solander) J.V. Lamouroux e Hypnea spinella (C. Agardh) Kütz.) contribuíram com 5 a $20 \%$ de biomassa. Esses resultados mostraram a existência de distúrbios, provavelmente decorrentes das dragagens para aumento do canal de navegação para o Porto de Sepetiba, além da entrada de frentes meteorológicas. Apesar da avaliação do potencial invasivo da espécie exótica Kappaphycus alvarezii (Doty) Doty ex Silva ter sido negativo neste período, se recomenda que o seu monitoramento seja permanente.

Palavras-chave: assembléia fitobentônica, baía de Sepetiba, ilha da Marambaia, monitoramento ambiental

ABSTRACT - (Characterization of the phytobenthic assemblage at Kutuca beach, Marambaia island, Sepetiba bay, Rio de Janeiro State, Brazil). Due to potential environmental problems in Sepetiba Bay, it is necessary to identify sites for environmental monitoring. Kutuca Beach was chosen for this purpose since community structure data from collections in 1999 detected high diversity. Over a period of 21 months, 63 taxa were collected (Chlorophyta, 22\%; Ochrophyta, 16\%; Rhodophyta, 62\%). The destructive sampling used six random plots $(25 \times 25 \mathrm{~cm})$ in each of two 20-meter long lines horizontal to the rocky shore. When the 1999 results were compared with these, it was observed that biomass went from $490.9 \pm 201.2$ g.m $\mathrm{m}^{-2}$ to $199.57 \pm 29.33 \mathrm{~g} . \mathrm{m}^{-2}$, richness from $13.0 \pm 4.5$ to $5.06 \pm 1.72$, diversity $\mathrm{H}^{\prime}=2.2 \pm 0.41$ to $\mathrm{H}^{\prime}=1.3 \pm 0.39$ and equitability $\mathrm{J}^{\prime}=0.65 \pm 0.06$ to $\mathrm{J}^{\prime}=0.55 \pm 0.17$. Four taxa (Sargassum spp., Caulerpa sertularioides (S.G. Gmel.) M. Howe, Hypnea musciformis (Wulfen in Jacquin) J.V. Lamour. and Gracilaria cervicornis (Turner) J. Agardh) contributed $15 \%$ to $33 \%$ of the biomass, while in 1999, eight taxa (Caulerpa sertularioides, Dictyopteris delicatula J.V. Lamour., Gracilaria cervicornis, Sargassum spp., Codium taylorii P.C. Silva, Padina gymnospora (Kützing) Sonder, Galaxaura marginata (Ellis \& Solander) J.V. Lamouroux and Hypnea spinella (C. Agardh) Kütz.) contributed 5\% to $20 \%$ of the biomass. These results showed the existence of disturbance that probably is a consequence of dredging to increase the navigation channel to Sepetiba Port, as well as the entrance of cold fronts. In spite of fact that the invasive potential of the exotic species Kappaphycus alvarezii (Doty) Doty ex Silva was assessed as negative during this period, permanent monitoring of this species is recommended.

Key words: environmental monitoring, Marambaia island, phytobenthic assemblage, Sepetiba bay

\section{Introdução}

Os municípios que abrangem a baía de Sepetiba são caracterizados por grande atividade pesqueira e turística. Esta baía está localizada num dos maiores pólos industriais do Estado do Rio de Janeiro (SEAMADS
2001). Consequientemente, esta região é propícia à poluição ambiental proveniente de efluentes líquidos, que ocasionam a contaminação, por metais pesados oriundos de indústrias e de poluição orgânica acarretada pelos efluentes domésticos (SEAMADS 2001; Amado Filho et al. 2003).

\footnotetext{
1 Instituto de Pesquisas Jardim Botânico do Rio de Janeiro/Ministério do Meio Ambiente, Rua Pacheco Leão 915, 22460-030 Rio de Janeiro, RJ, Brasil

2 Autor para correspondência: rreis@jbrj.gov.br
} 
Além disso, existe o impacto causado pelo aumento de embarcações para o Porto de Sepetiba e as dragagens para manutenção e ampliação de seus canais de navegação (CDRJ 2005) e do potencial impacto causado pela água de lastro (Széchy et al. 2005).

Outro potencial impacto são os cultivos comerciais da alga exótica Kappaphycus alvarezii (Doty) Doty ex P.C. Silva. Em 2003, foi implantado na baía da Marambaia, inserida na baía de Sepetiba, o maior cultivo brasileiro desta espécie (Reis et al. 2006; 2007), posteriormente outros foram introduzidos nesta baía (observação pessoal).

O conhecimento sobre a estrutura de assembléias fitobentônicas é uma importante ferramenta para avaliação ambiental (Brito et al. 2002). Para isto, existe a necessidade de se estabelecer áreas controles para monitoramentos ambientais, nos quais sítios que possuam estudos pretéritos são os mais indicados para esta atividade. Na caracterização da estrutura da comunidade da baía de Sepetiba, realizada por Amado Filho et al. (2003), foi constatado que, dentre os cinco sítios amostrados, a praia do Kutuca foi o que apresentou maior riqueza específica e diversidade. Esta praia se localiza entre um dos canais mais importantes de entrada de correntes da baía de Sepetiba, entre a Ponta dos Castelhanos, na Ilha Grande e a Ponta Grossa - na ilha da Marambaia. A circulação da água desta baía é regida pelo fluxo e refluxo da maré e a entrada de água do mar ocorre através de passagens e canais existentes entre o continente e as ilhas de Itacuruçá, Jaguanum e Pombeba (SEMADS 2001). Por esses fatos, este local foi escolhido para o monitoramento ambiental desta baía, através da avaliação de sua assembléia fitobentônica e da averiguação sobre o estabelecimento de espécies exóticas, via água de lastro ou pela dispersão de mudas de Kappaphycus alvarezii cultivadas próximas a esta praia.

\section{Material e métodos}

A baía de Sepetiba se localiza ao sul do Estado do Rio de Janeiro e possui perímetro de $123 \mathrm{~km}$ (Silva \& Araújo 2003). Este estudo foi desenvolvido na ilha da Marambaia, no costão rochoso esquerdo da praia do Kutuca, Município de Mangaratiba (4359'35"W e $23^{\circ} 04^{\prime} 00$ '’S, Fig. 1).

As coletas foram realizadas a partir de novembro/2003 até julho/2005. Foi adotada a amostragem destrutiva e estabelecido o número mínimo de quadrados para a amostragem através da curva da média acumulada de quadrados (De Wreede 1985). Seis quadrados aleatórios foram colocados ao longo de linhas horizontais ao costão. As linhas foram colocadas a 1 e $2 \mathrm{~m}$ de profundidade da maré baixa de sizígia, através de

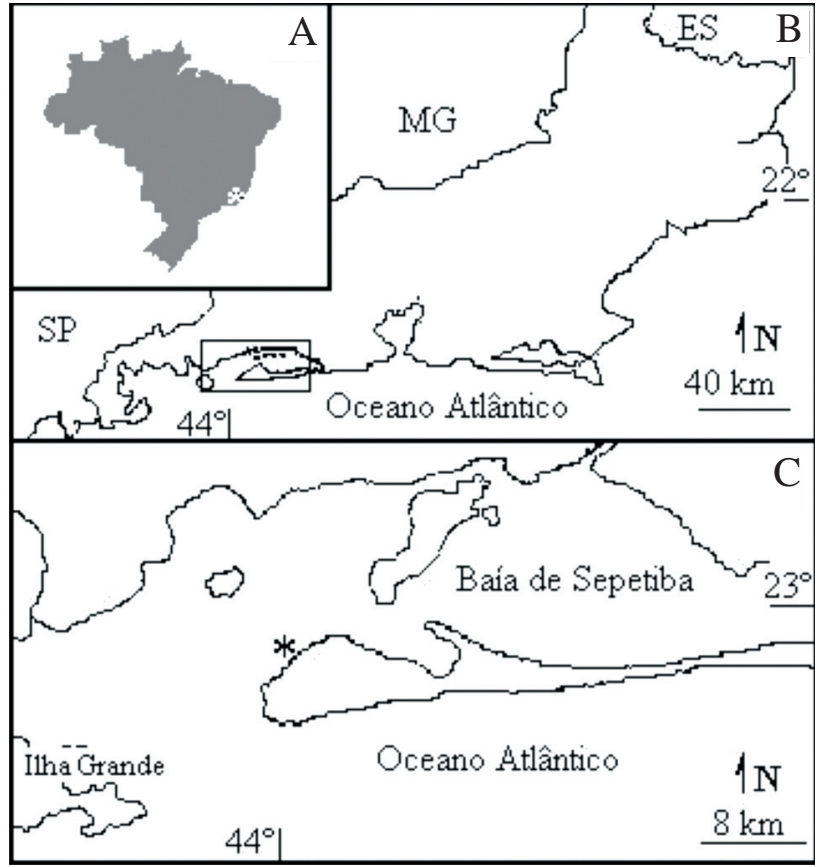

Figura 1. A. Mapa do Brasil, asterisco branco indica a localização do Estado do Rio de Janeiro. B. Estado do Rio de Janeiro com a baía de Sepetiba em destaque. C. Asterisco escuro indica a localização da Praia do Kutuca.

mergulho autônomo. Amostragens qualitativas também foram realizadas. A classificação taxonômica e a nomenclatura escolhida seguiram a adotada por Wynne (2005) e na ausência de táxon nesta citação foi utilizada a base de dados do Guyri \& Guiry (2008).

O material coletado foi fixado em solução de formaldeido a $4 \%$ e água do mar para posterior identificação e quantificação da massa seca constante obtida em estufa a $60{ }^{\circ} \mathrm{C}$. Os táxons com biomassa inferior a $0,001 \mathrm{~g}$ (massa seca) foram agrupados e denominados como outros. Também foram classificados em grupos morfofuncionais, com base em características morfológicas, seguindo a classificação adotada por Littler \& Littler (1984) e Steneck \& Dethier (1994), que os correlacionam com suas características ecológicas.

Foram avaliadas: a riqueza de espécies, a biomassa e a diversidade. Esta última através dos índices de diversidade - H' (Shannon \& Weaver) e de equitabilidade - J' (Pielou) (Clarke \& Warwick 1994; Brower et al. 1997). Os organismos foram quantificados através do percentual de sua biomassa em relação à biomassa total dos organismos, usando a fórmula, Percentual de Contribuição $=$ (média da massa seca do organismo/média da massa total dos organismos) $\times 100$ (Amado Filho et al. 2003). Foram denominados como organismos dominantes àqueles com Percentual de Contribuição acima de $5 \%$ e como outros os organismos com valores 
inferiores. As espécies de Sargassum foram agrupadas em Sargassum spp.

A temperatura e a salinidade da água do mar da praia do Kutuca foram registradas diariamente, com o auxílio de termômetro de mercúrio e refratômetro manual, respectivamente, enquanto que a velocidade dos ventos, precipitação da água e nebulosidade foram obtidas através de dados coletados na Base Aérea de Santa Cruz,

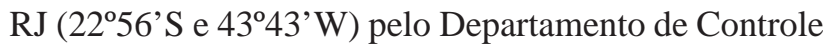
do Espaço Aéreo/Serviços Regionais de Proteção ao Vôo da Aeronáutica - DECEA/SRPV (Tab. 1).

A normalidade dos dados foi testada pelo teste de Shapiro-Wilk e a heterogeneidade pelo teste de Cochran. Dados heterogêneos passaram por transformação logarítmica. As diferenças entre as médias da diversidade (H' e J') foram averiguadas pela análise de variância unifatorial (ANOVA). As diferenças entre elas foram separadas pelo teste de Tukey e representadas em gráficos como média \pm desvio padrão. As medianas das biomassas e das riquezas foram testadas através do teste de Kruskal-Wallis e graficamente representadas em mediana, quartil (25-75\%) e valores mínimos e máximos (Min-Máx) (Zar 1996). As biomassas totais de dois períodos estudados (1999 e 2003-2005) foram comparadas pelo t-teste e a riqueza pelo teste de MannWhitney. O intervalo de confiança para os testes de significância foi de $95 \%(p=0,05)$.

\section{Resultados e discussão}

Foram identificados na análise qualitativa 63 táxons, pertencentes a Chlorophyta (14 táxons $=22 \%)$; Ochrophyta (10 táxons $=16 \%$ ) e Rhodophyta (39 táxons $=62 \%$ ). Estes estão citados na Tab. 2 , junto com os 63 táxons encontrados nesta praia em 1999, por Amado Filho et al. (2003), correspondentes as Chlorophyta (10 táxons $=16 \%)$; Ochrophyta (10 táxons $=16 \%)$ e Rhodophyta (43 táxons $=68 \%$ ). Novas ocorrências não foram encontradas em relação aos 235 táxons de algas listados para a baía de Sepetiba, em levantamento de dados pretéritos, a saber: 151 Rhodophyta, 35 Ochrophyta, 49 Chlorophyta (Amado Filho \& Marins 2004).

Tanto na amostragem qualitativa quanto na quantitativa, não foram encontradas mudas da alga exótica Kappaphycus alvarezii. Esta alga vem sendo cultivada comercialmente na baía da Marambaia, localizada cerca de $20 \mathrm{~km}$ desta praia. No período amostrado (novembro/2003 até julho/2005), o cultivo possuía aproximadamente 50 balsas flutuantes, com $150 \mathrm{~m}$ de comprimento e $3 \mathrm{~m}$ de largura (Reis et al. 2007). A praia do Kutuca está próxima a um dos canais de correntes mais importantes da baía de Sepetiba (SEMADS 2001). Desta maneira, ao avaliar a ausência do estabelecimento de mudas, se considerou um

Tabela 1. Média \pm desvio padrão da temperatura e da salinidade da água do mar na praia do Kutuca, ilha da Marambaia, baía de Sepetiba, RJ, Brasil, e da velocidade dos ventos e nebulosidade e soma da precipitação de água cedidos pela Base Aérea de Santa Cruz, RJ, Brasil (2256'S e $\left.43^{\circ} 43^{\prime} \mathrm{W}\right)$ pelo Departamento de Controle do Espaço Aéreo/Serviços Regionais de Proteção ao Vôo da Aeronáutica - DECEA/SRPV.

\begin{tabular}{|c|c|c|c|c|c|}
\hline Data & $\begin{array}{c}\text { Temperatura } \\
\left({ }^{\circ} \mathrm{C}\right)\end{array}$ & Salinidade & $\begin{array}{l}\text { Precipitação } \\
\left(\mathrm{mm} \cdot \mathrm{mes}^{-1}\right)\end{array}$ & $\begin{array}{l}\text { Velocidade do vento } \\
\text { (nós) }\end{array}$ & $\begin{array}{c}\text { Nebulosidade } \\
(0-8)\end{array}$ \\
\hline Novembro 2003 & & & 117,7 & $8,2 \pm 4,6$ & $5,3 \pm 2,3$ \\
\hline Dezembro 2003 & $25,2 \pm 2,40$ & $34,7 \pm 0,6$ & 88,5 & $6,8 \pm 4,2$ & $5,3 \pm 2,4$ \\
\hline Janeiro 2004 & $25,6 \pm 1,90$ & $32,6 \pm 1,2$ & 163,2 & $6,6 \pm 3,9$ & $5,0 \pm 2,6$ \\
\hline Fevereiro 2004 & $27,1 \pm 1,30$ & $31,9 \pm 1,5$ & 141,5 & $5,9 \pm 3,5$ & $5,1 \pm 2,7$ \\
\hline Março 2004 & $27,5 \pm 1,70$ & $32,9 \pm 1,7$ & 80,8 & $6,4 \pm 3,9$ & $4,5 \pm 2,4$ \\
\hline Abril 2004 & $24,3 \pm 1,10$ & $33,1 \pm 1,6$ & 93,4 & $5,5 \pm 3,6$ & $4,8 \pm 2,3$ \\
\hline Maio 2004 & $24,0 \pm 1,30$ & $33,6 \pm 1,6$ & 90,5 & $4,9 \pm 3,5$ & $4,6 \pm 2,7$ \\
\hline Junho 2004 & $24,4 \pm 1,70$ & $34,2 \pm 1,1$ & 85,9 & $5,9 \pm 4,2$ & $2,8 \pm 3,0$ \\
\hline Julho 2004 & $24,6 \pm 1,30$ & $32,9 \pm 2,1$ & 124,9 & $5,6 \pm 4,5$ & $3,3 \pm 3,2$ \\
\hline Agosto 2004 & $25,4 \pm 1,40$ & $36,6 \pm 1,3$ & 10,1 & $5,5 \pm 4,5$ & $2,9 \pm 3,0$ \\
\hline Setembro 2004 & $23,9 \pm 1,40$ & $35,5 \pm 1,1$ & 26,2 & $6,9 \pm 4,9$ & $2,9 \pm 3,2$ \\
\hline Outubro 2004 & $25,8 \pm 1,80$ & $35,3 \pm 1,2$ & 92,6 & $7,1 \pm 4,9$ & $5,4 \pm 2,7$ \\
\hline Novembro 2004 & $27,2 \pm 1,80$ & $34,6 \pm 1,4$ & 115,1 & $7,0 \pm 4,7$ & $5,4 \pm 2,7$ \\
\hline Dezembro 2004 & $25,5 \pm 1,56$ & $33,8 \pm 2,3$ & 117,2 & $6,2 \pm 4,2$ & $5,7 \pm 2,1$ \\
\hline Janeiro 2005 & $26,0 \pm 2,60$ & $33,8 \pm 1,2$ & 93,4 & $6,5 \pm 3,6$ & $5,9 \pm 2,1$ \\
\hline Fevereiro 2005 & $26,3 \pm 2,10$ & $30,4 \pm 1,3$ & 122,2 & $6,7 \pm 3,8$ & $4,4 \pm 2,2$ \\
\hline Março 2005 & $24,8 \pm 3,40$ & $29,3 \pm 1,9$ & 411,4 & $5,8 \pm 4,0$ & $4,7 \pm 2,7$ \\
\hline Abril 2005 & $24,6 \pm 3,70$ & $32,2 \pm 1,1$ & 119,5 & $6,4 \pm 4,0$ & $4,0 \pm 2,9$ \\
\hline Maio 2005 & $24,3 \pm 2,50$ & $34,4 \pm 0,7$ & 88,1 & $5,6 \pm 3,6$ & $3,0 \pm 3,1$ \\
\hline Junho 2005 & $25,2 \pm 2,40$ & $34,3 \pm 0,8$ & 52,5 & $5,5 \pm 1,4$ & $2,1 \pm 2,5$ \\
\hline Julho 2005 & $25,6 \pm 2,00$ & $34,7 \pm 0,6$ & 67,7 & $5,3 \pm 2,1$ & $3,1 \pm 2,9$ \\
\hline
\end{tabular}


Tabela 2. Táxons coletados na praia do Kutuca, ilha da Marambaia, baía de Sepetiba, RJ, Brasil, no período entre novembro/2003 a julho/2005 e por Amado Filho et al. (2003) em 1999.

\begin{tabular}{|c|c|c|c|c|c|}
\hline Táxons & 003-2005 & 1999 & Táxons & $003-2005$ & 1999 \\
\hline \multicolumn{3}{|l|}{ CHLOROPHYTA } & Ceramium sp. 2 & - & + \\
\hline Bryopsis pennata J. V. Lamour. & + & - & Ceramium sp. 3 & + & - \\
\hline B. plumosa (Huds.) C. Agardh & - & + & Champia parvula (C. Agardh) Harv. & + & + \\
\hline Caulerpa fastigiata Mont. & + & + & C. salicornioides Harv. & - & + \\
\hline \multirow{2}{*}{$\begin{array}{l}\text { C. racemosa var. Peltata } \\
\text { (J.V. Lamouroux) Eubank }\end{array}$} & \multirow[t]{2}{*}{+} & \multirow[t]{2}{*}{-} & Chondracanthus acicularis (Roth) Fredericq & + & + \\
\hline & & & C. teedei (Mertens ex Roth) Fredericq & + & - \\
\hline C. sertularioides (S.G. Gmel.) M. Howe & + & + & \multirow{2}{*}{\multicolumn{2}{|c|}{$\begin{array}{l}\text { Cryptonemia delicatula A.B. Joly \& } \\
\text { Cordeiro in Joly et al. }\end{array}$}} & \multirow[t]{2}{*}{-} \\
\hline Chaetomorpha antennina (Bory) Kütz. & + & - & & & \\
\hline Cladophora brasiliana G. Martens & - & + & Dasya brasiliensis E.C. Oliveira \& Y. Braga & + & + \\
\hline C. coelothrix Kütz. & - & + & \multirow{2}{*}{\multicolumn{2}{|c|}{$\begin{array}{l}\text { Dichotomaria marginata (J. Ellis \& Sol.) } \\
\text { Lamark }\end{array}$}} & \multirow[t]{2}{*}{+} \\
\hline C. capensis (C. Agardh) De Toni & + & + & & & \\
\hline C. vagabunda (L.) C. Hoek & + & - & Erythrotrichia carnea (Dillwyn) J. Agardh & + & - \\
\hline $\begin{array}{l}\text { Cladophoropsis membranaceae } \\
\text { (C. Agardh) Börgesen }\end{array}$ & + & - & $\begin{array}{l}\text { Gelidiopsis planicaulis (W.R. Taylor) } \\
\text { W.R. Taylor }\end{array}$ & + & + \\
\hline Codium decorticatum (Woodw.) M. Howe & + & + & G. variabilis (Grev. ex J. Agardh) F. Schmitz & + & + \\
\hline C. taylorii P.C. Silva & + & + & Gelidium crinale (Turner) Gaillon & + & + \\
\hline Derbesia marina (Lyngb.) Solier & + & - & G. pusillum (Stackh.) Le Jolis var. pusillum & + & + \\
\hline Ulva flexиosa Wulfen sub.flexиosa & + & - & Gracilaria cerviconis (Turner) J. Agardh & + & + \\
\hline U. lactuca $\mathrm{L}$ & + & + & \multirow{2}{*}{\multicolumn{2}{|c|}{$\begin{array}{l}\text { Gracilariopsis tenuifrons (C.J. Bird \& E.C } \\
\text { Oliveira) Fredericq \& Hommersand }\end{array}$}} & \multirow[t]{2}{*}{+} \\
\hline U. rigida C. Agardh & + & + & & & \\
\hline Subtotal & 14 & 10 & Grateloupia cuneifolia J. Agardh & - & + \\
\hline \multicolumn{3}{|l|}{ OCHROPHYTA } & G. filicina (J.V. Lamour.) C. Agardh & + & + \\
\hline \multirow{4}{*}{$\begin{array}{l}\text { Bachelotia antillarum (Grunow) Gerloff } \\
\text { Chnoospora mínima (K. Hering) Papenf. } \\
\text { Colpomenia sinuosa (Roth) Derdès \& Solier } \\
\text { Dictyopteris delicatula J.V. Lamour. }\end{array}$} & + & - & Gymnogongrus griffithsiae (Turner) Mart. & + & - \\
\hline & + & - & Halymenia elongata C. Agardh & - & + \\
\hline & \multirow[t]{2}{*}{$\mathrm{r}+$} & + & H. rosea Howe \& W.R. Taylor & + & + \\
\hline & & + & Herposiphonia secunda (C. Agardh) Ambronn & + & - \\
\hline Dictyota ciliolata Sond. ex Kütz. & $\begin{array}{l}+ \\
+\end{array}$ & + & H. tenella (C. Agardh) Ambronn & + & + \\
\hline Hincksia mitchelliae (Harv.) P.C. Silva & $\begin{array}{l}+ \\
+\end{array}$ & $\begin{array}{l}+ \\
+\end{array}$ & Hydrpuntia caudata (J.Agardh) Gurgel \& & + & + \\
\hline Padina gymnospora (Kützing) Sonder & + & + & Fredericq & & \\
\hline P. antillarum (Kütz) Picc. & - & + & Hypnea musciformis (Wulfen in Jacquin) & + & + \\
\hline Sargassum cymosum C. Agardh & + & + & J.V. Lamour. & & \\
\hline $\begin{array}{l}\text { Sargassum cymosum } \mathrm{C} \text {. Agardn } \\
\text { var. cymosum }\end{array}$ & + & + & H. spinella (C. Agardh) Kütz. & + & + \\
\hline S. stenophyllum Mart. & + & + & Jania adhaerens J.V. Lamour. & + & + \\
\hline Spatoglossum schroederi (C. Agardh) Kütz. & - & + & Neosiphonia ferulacea (Surh ex J. Agardh) & + & + \\
\hline Sphacelaria tribuloides Menegh. & + & + & S.M. Guim. \& M.T.Fujii & & \\
\hline Subtotal & 10 & 10 & N. sphaerocarpa (Börgesen) M.S. Kim \& & - & + \\
\hline RHODOPHYTA & & & N. tongatensis (Harv. ex Kütz) M.S. Kim \& & - & + \\
\hline Acanthophora spicifera (Vahl) Börgesen & + & - & I.K. Lee & & \\
\hline $\begin{array}{l}\text { Acrochaetium microscopicum } \\
\text { (Nägeli ex Kütz.) Nägeli }\end{array}$ & + & - & $\begin{array}{l}\text { Polysiphonia denudata (Dillwyn) Grev. ex } \\
\text { Harv. in Hook. }\end{array}$ & + & - \\
\hline $\begin{array}{c}\text { Aglaothamnion uruguayense (W.R. Taylor) } \\
\text { N. Aponte, D.L. Ballant \& J.N. Norris }\end{array}$ & + & + & $\begin{array}{l}\text { P. scopulorum Harv. var. villum (J. Agardh) } \\
\text { Hollenb. }\end{array}$ & - & + \\
\hline Amphiroa beauvoisii J.V. Lamour. & - & + & P. subtilissima Mont. & + & + \\
\hline A. brasiliana Decne. & + & - & Pterocladiella caerulescens (Kütz.) Santel. \& & - & + \\
\hline A. fragilissima (L.) J.V. Lamour. & - & + & Hommers. & & \\
\hline $\begin{array}{l}\text { Bostrychia radicans (Mont.) } \\
\text { Mont. in Orbigny }\end{array}$ & + & + & $\begin{array}{l}\text { Pterosiphonia parasitica (Huds.) Falkenb. } \\
\text { var. australis A.B. Joly \& Cord.- Mar. }\end{array}$ & + & + \\
\hline B. tenella (J.V. Lamour.) J. Agardh & + & + & P. pennata (C. Agardh) Falkenb. & + & + \\
\hline Bryocladia cuspidata (J. Agardh) De Toni & + & - & Rhodymenia pseudopalmata (J. V. Lamour.) & + & + \\
\hline B. thyrsigera (J. Agardh) & + & + & P.C. Silva & & \\
\hline F. Schmitz in Falkenb. & & & Sahlingia subintegra (Rosenv.) Kornmann & + & + \\
\hline Centroceras clavulatum (C. Agardh in & + & + & Solieria filiformis (Kütz.) P.W. Gabrielson & - & + \\
\hline Kunth) Mont. in Durieu de Maisonneuve & & & Spyridia filamentosa (Wulfen) Harv. in Hook. & + & + \\
\hline Ceramium diaphanum (Lightf.) Roth & - & + & Subtotal & 39 & 43 \\
\hline C. flaccidum (Kütz.) Ardiss. & - & + & & & \\
\hline Ceramium sp.1 & - & + & Total geral & 63 & 63 \\
\hline
\end{tabular}


resultado positivo para a permissão do cultivo desta espécie na baía de Sepetiba, atualmente em análise no Instituto do Meio Ambiente e dos Recursos Naturais Renováveis - IBAMA (Instrução Normativa do IBAMA n. 165 de 17 de julho de 2007).

Ao longo dos 21 meses de coleta, através da amostragem quantitativa, foi observado que a riqueza, a biomassa, os índices de diversidade e de equitabilidade variaram ao longo do período estudado, assim como os percentuais de cobertura dos táxons com percentuais acima de $5 \%$ (Tab. 3, Fig. 2, 3). Ao comparar esses valores com os valores encontrados em 1999, constatou-se alteração na estrutura da assembléia fitobentônica (Tab. 4). A riqueza ( $\mathrm{Z}=2,3$ $\mathrm{p}=0,03, \mathrm{n}=4)$ e a biomassa (t-teste $=2,73, \mathrm{p}=0,03)$ diminuíram, assim como ocorreu uma tendência de diminuição na diversidade com distribuição heterogênea das espécies.

Tabela 3. Teste de Kruskal-Wallis (H') para a riqueza específica e para biomassa e Análise de Variância unifatorial - ANOVA(F) para os índices de diversidade de Shanon e de equitabilidade de Pielou, relativos ao período da primavera de 2003 ao inverno de 2005 .

\begin{tabular}{lcc}
\hline Tipo de teste & Resultado & P level \\
\hline Riqueza de espécies & $\mathrm{H}^{\prime}=26,67$ & $<0,001$ \\
Biomassa & $\mathrm{H}^{\prime}=30,31$ & $<0,001$ \\
Diversidade de Shanon & $\mathrm{F}=5,58$ & $<0,001$ \\
Equitabilidade de Pielou & $\mathrm{F}=5,54$ & $<0,001$ \\
\hline
\end{tabular}

Observou-se uma redução de oito para quatro táxons (considerando Percentual de Contribuição acima de 5\%) de 1999 (Amado Filho et al. 2003) para o atual estudo (coletas de 2003-2005). Em 1999 foram encontradas Caulerpa sertularioides (16\%), Dictyopteris delicatula (15\%), Gracilaria cervicornis (13\%), Sargassum spp. (10\%), Codium taylorii (9\%), Padina gymnospora (9\%), Galaxaura marginata (5\%) e Hypnea spinella (5\%) e no presente estudo 2003 a 2005, Sargassum spp. (33\%), Caulerpa sertularioides (15\%), Hypnea musciformis (18\%) e Gracilaria cervicornis (19\%) (Fig. 4).

Silveira (2004) comenta que ambientes não perturbados são caracterizados por alta diversidade e equitabilidade e que a diminuição na diversidade e o aumento na dominância sejam ocasionados pela perda de organismos mais sensíveis e aumento na abundância de organismos mais tolerantes.

Foi constatado um maior Percentual de Contribuição de algas do grupo corticadas e coriáceas (53 e 33\%), quando comparado com o período de 1999 (47 e 10\%). Estes grupos possuem alargamento do córtex, com paredes celulares mais espessas do que o observado para as algas corticadas foliosas, como as espécies de Padina,

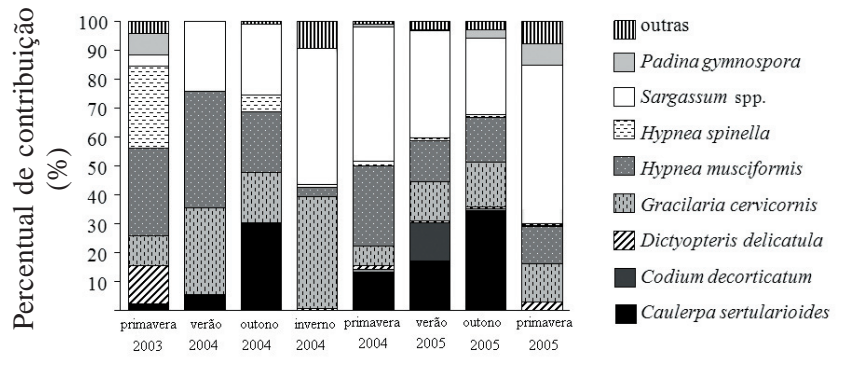

Figura 2. Percentual de Contribuição dos táxons encontrados na praia do Kutuca, ilha da Marambaia, RJ, Brasil, entre a primavera de 2005 e o inverno de 2005

Dictyopteris e Dictyota. Estas características tornam essas algas mais resistentes a distúrbios físicos e biológicos (Steneck \& Dethier 1994). No período de novembro/2003 até julho/2005, as algas corticadas foliosas não foram encontradas em percentuais acima de 5\%, representadas principalmente por Dictyopteris delicatula (3\%), enquanto que em 1999, dos $24 \%$ de algas corticadas foliosas, $15 \%$ foram de Dictyopteris delicatula (Fig. 6). Este fato deve ser investigado, uma vez que, esta espécie é uma epífita de Sargassum (Széchy \& Paula 1997), que compete com Hypnea musciformis e Hypnea spinella - corticadas (Reis \& YoneshigueValentin 1998), que aumentaram de $1 \%$ para $13 \%$.

O Percentual de Contribuição das espécies de Sargassum (algas coriáceas) aumentou em relação ao encontrado em 1999 e, geralmente, com maiores percentuais a cada coleta. Após o inverno de 2004, se mantiveram próximos a $40 \%$ (Fig. 2). Este gênero é encontrado em abundância nos costões rochosos da região sudeste brasileira (Széchy \& Paula 2000; Falcão \& Széchy 2005). O padrão estrutural do banco estudado se assemelha ao descrito para ambientes protegidos e moderado, onde as algas corticadas são representadas principalmente por espécies de Hypnea (Széchy \& Paula 2000), aqui com representação de cerca de $20 \%$. Esta dominância não é observada em locais com forte movimentação de água e poluição (Falcão \& Széchy 2005).

Sugere-se que a mudança na diversidade e na dominância, principalmente por espécies de Sargassum, foram ocasionadas por distúrbios físicos, como o aparente assoreamento dos matacões nesta praia. Provavelmente, decorrente das dragagens para manutenção do canal de navegação em direção ao Porto de Sepetiba (CDRJ 2005), que propiciaram mudanças no fundo desta baía, com a formação de bancos de sedimentos próximos à praia estudada (observação pessoal e relato de pescadores), com consequiências no hidrodinamismo local. Além disso, o predomínio de ventos, no quadrante norte-leste, aumentou a turbidez 
da água, fato este observado por Reis et al. (2006). Estas mudanças devem ter desfavorecido o crescimento das corticadas foliosas.

Outro fato a ser investigado foi a tendência da diversidade e da equitabilidade terem sido maiores em março/2005 (Fig. 3), coincidindo com o aumento na precipitação, com consequiente diminuição da salinidade, que ocorreram nesta época e com o aumento da temperatura da água do mar. A salinidade (Kruskal-Wallis, $\mathrm{H}=276,98, \mathrm{p}<0,001)$ e a temperatura (Kruskal-Wallis, $\mathrm{H}=113,13, \mathrm{p}<0,001)$ variaram ao longo do tempo.
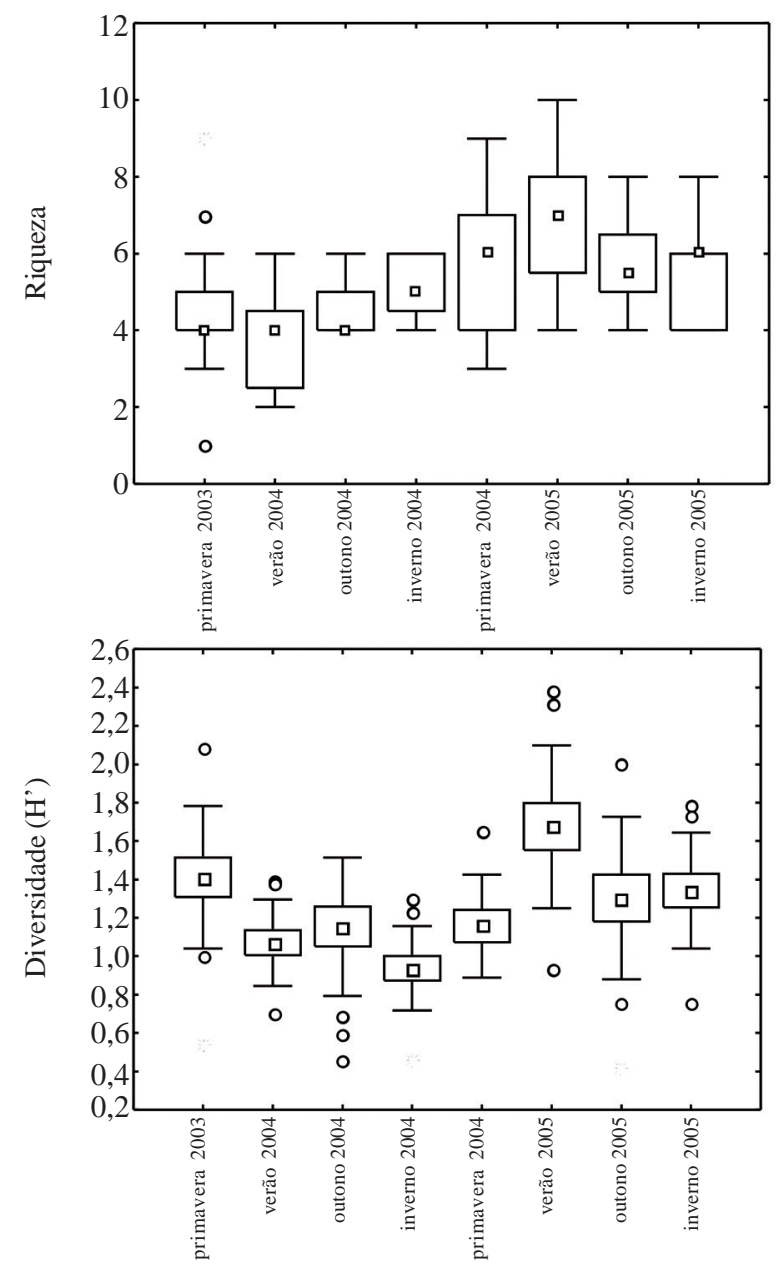

Concluindo, foi observada mudança na estrutura da assembléia fitobentônica da praia do Kutuca no período de novembro/2003 a julho/2005 em relação ao de 1999, com menor riqueza (amostragem quantitativa), biomassa e diversidade e maior dominância de espécies. O número de táxons passou de oito para quatro táxons (agrupando as espécies de Sargassum e considerando algas com percentual de contribuição acima de 5\%). Sugere-se que esta mudança deve ter sido consequiência de distúrbios físicos locais. Os possíveis distúrbios foram: as dragagens para o Porto de Sepetiba que ocasionaram
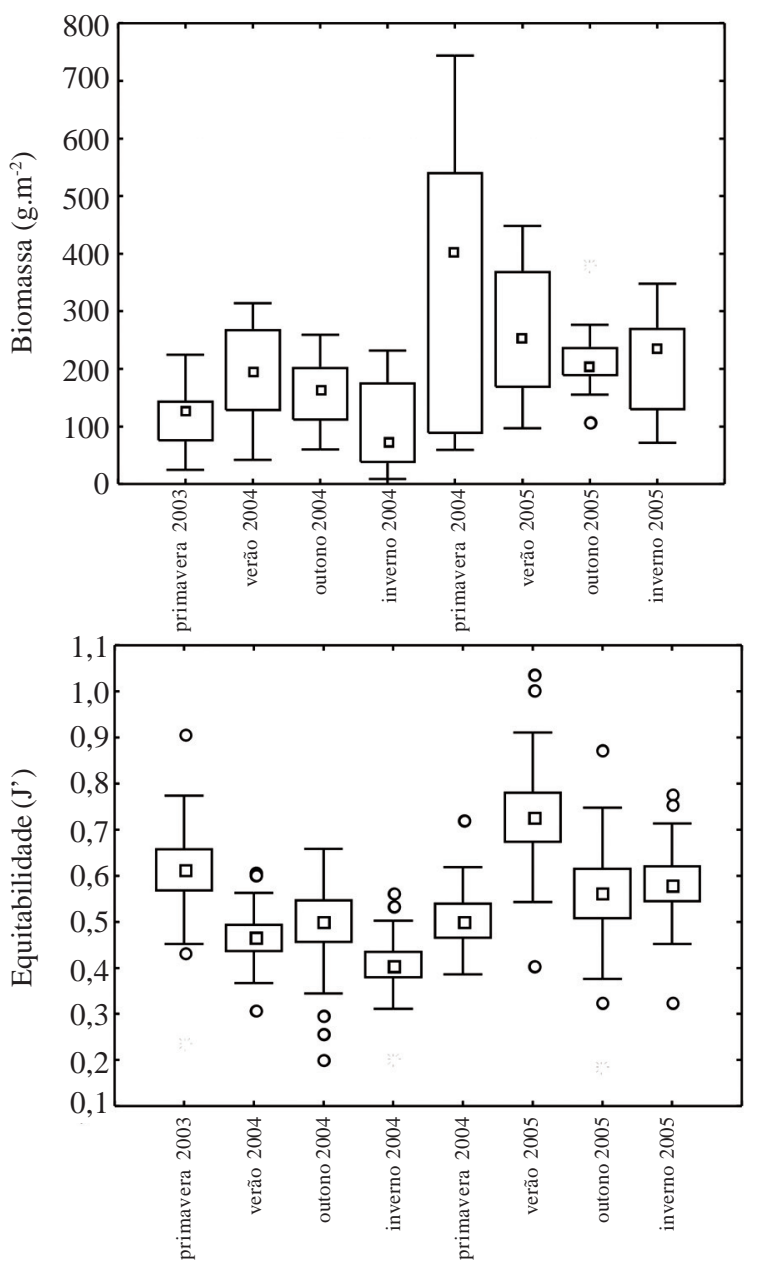

Figura 3. Representação gráfica da riqueza, biomassa, diversidade e equitabilidade das algas na praia do Kutuca, RJ, Brasil, no período entre a primavera de 2005 e o inverno de 2005. Nas figuras sobre a riqueza e a biomassa, o quadrado maior indica a mediana, o retângulo a faixa entre $25-75 \%$ dos dados. Nas figuras sobre diversidade e equitabilidade, o quadrado menor indica a média e o maior o erro padrão, os traços mostram o desvio padrão. Em todas as figuras o círculo indica os outilier e o asterisco os valores extremos.

Tabela 4. Média \pm desvio padrão da riqueza de espécies, biomassa e índices de diversidade e de equitabilidade das algas da praia do Kutuca, RJ, Brasil, no período entre novembro/2003 a julho/2005.

\begin{tabular}{lcccc}
\hline Período & Riqueza de espécies & $\begin{array}{c}\text { Biomassa } \\
\left(\mathrm{g} \text { massa seca. } \mathrm{m}^{-2}\right)\end{array}$ & Diversidade & Equitabilidade \\
\hline 1999 & $13,0 \pm 4,5$ & $490,9 \pm 201,2$ & $2,2 \pm 0,41$ & $0,65 \pm 0,06$ \\
$2003-2005$ & $5,06 \pm 1,72$ & $199,57 \pm 29,33$ & $1,3 \pm 0,39$ & $0,55 \pm 0,17$ \\
\hline
\end{tabular}




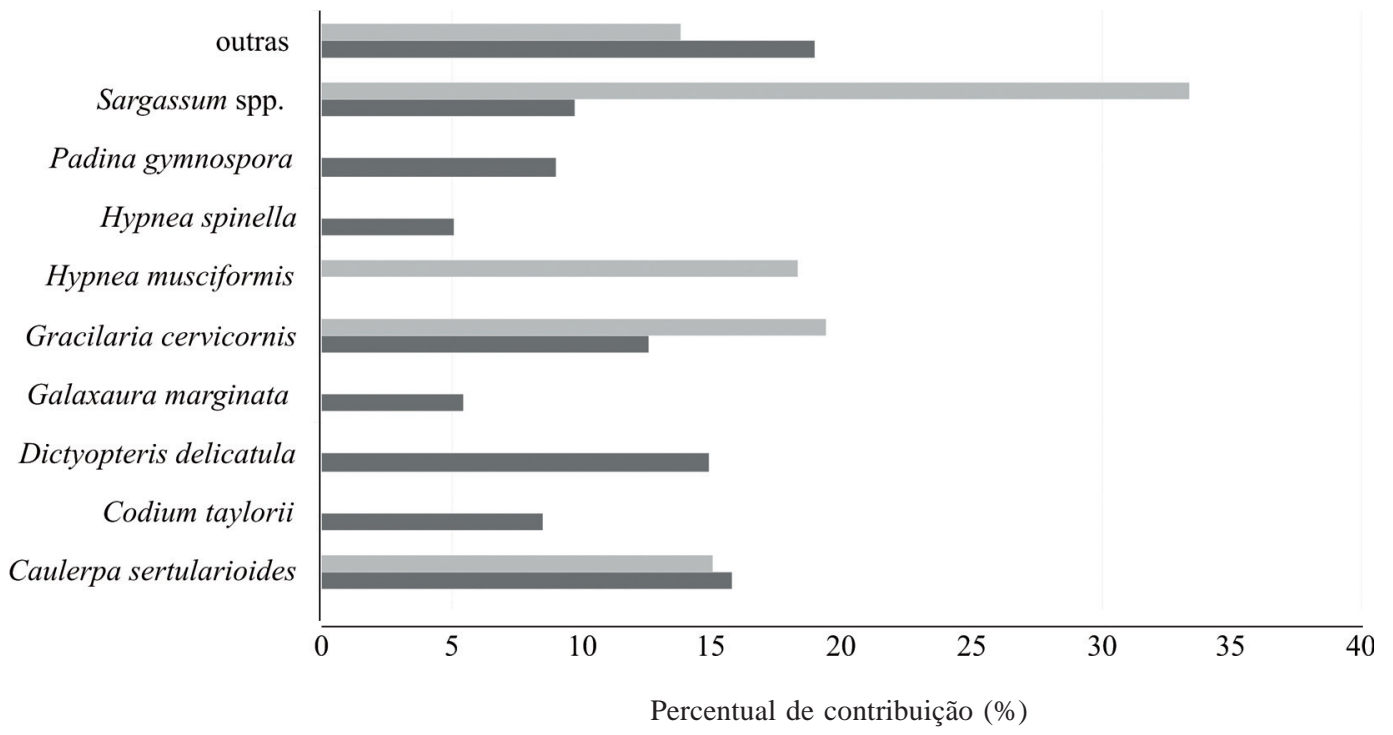

Figura 4. Percentual de Contribuição dos táxons mais representativos da praia do Kutuca, coletados em 1999 e no período entre novembro/2003 a julho/2005 ( $\mathbf{\square}=2003-2005 ; \quad=1999)$.

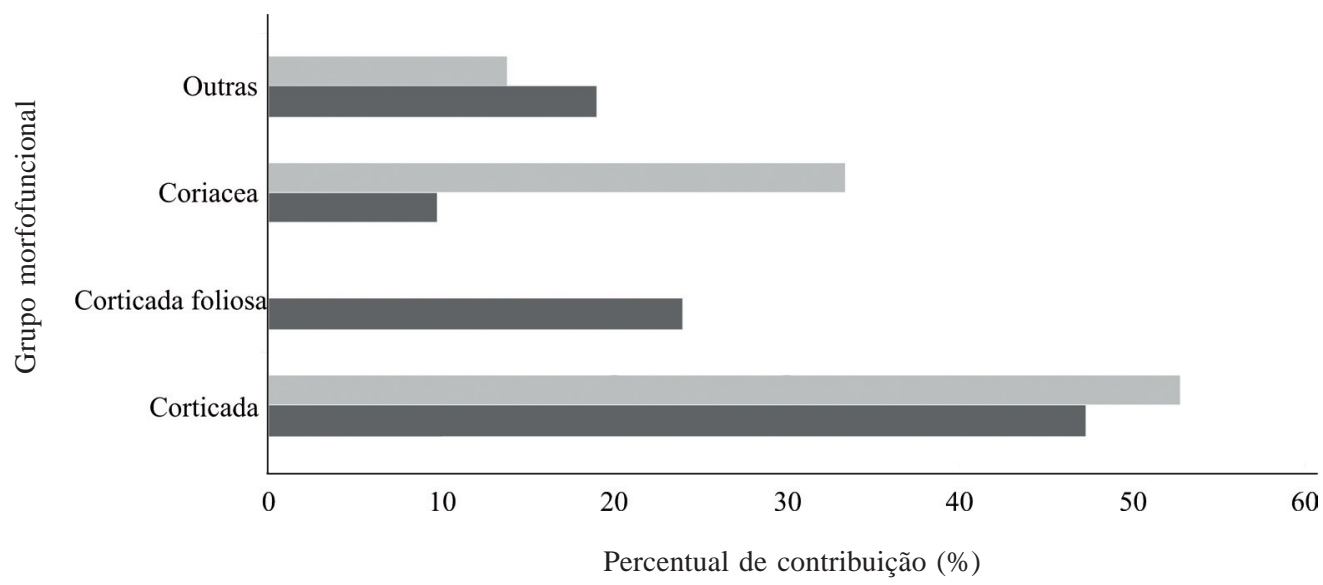

Figura 5. Percentual de Contribuição dos grupos morfo-funcionais encontrados na praia do Kutuca em 1999 e no período entre novembro/2003 a julho/2005 ( = 2003-2005; $\square=1999)$.

aumento dos bancos de sedimentos e alteração no hidrodinamismo da praia do Kutuca, com conseqüente assoreamento dos matacões desta praia. Outro distúrbio é a entrada de frentes meteorológicas, que aumentaram a turbidez da água. O potencial invasivo da espécie exótica Kappaphycus alvarezii foi negativo no período estudado, entretanto, é recomendado o seu monitoramento permanente na área.

\section{Agradecimentos}

À Fundação Carlos Chagas Filho de Amparo à Pesquisa do Estado do Rio de Janeiro, pelo apoio financeiro (E-26/170.191/2003 - APQ1 e E-26/152.303/ 2003, bolsa IC) e à Ana Paula dos Santos Miranda pelo auxílio na triagem e identificação das algas.

\section{Referências bibliográficas}

Amado Filho, G.M.; Barreto, M.B.B.; Marins, B.B.V.; Felix, C. \& Reis, R.P. 2003. Estrutura da comunidade fitobentônica do infralitoral da baía de Sepetiba, RJ, Brasil. Revista Brasileira de Botânica 26: 329-342.

Amado Filho, G.M. \& Marins, B.B.V. 2004. Fitobentos. Pp. 37-45. In: M.C. Villac; F.C. Fernandes, S. Jablonski, A.C. Leal Neto \& B.E. Coutinho (eds.). Biota da área sob influência do Porto de Sepetiba, Rio de Janeiro, Brasil. Levantamento de dados pretéritos. GlobalBallsat Water Management Programme.

Brower, J.E.; Zar, J.H. \& Von Ende, C. 1997. Field and laboratory methods for general ecology. McGraw-Hill.

Brito, L.V.R.; Széchy, M.T.M. \& Cassano, V. 2002. Levantamento taxonômico das macroalgas da zona das marés de costões rochosos adjacentes ao Terminal Marítimo Almirante Maximiano 
Fonseca, baía da Ilha Grande. Atlântica 24: 17-26.

CDRJ - Companhia Docas do Rio de Janeiro - Portobrás. [on line]. Disponível na Internet via www.url: http://www.portosrio.gov.br/ sepetiba/Setpor.htm (Acesso em: 5/agosto/2005).

Clarke, K.R. \& Warwick, R.M. 1994. Change in marine communities: an approach to statistical analysis and interpretation. Plymouth Marine Laboratory. Bourne Press Limited, Bournemouth.

De Wreede, R.E. 1985. Destructive (harvest) sampling. Pp.147-160. In: M.M. Littler \& D.S. Littler (eds.). Handbook of phycological methods: ecological field methods: Macroalgae. Cambridge, Cambridge University Press.

Falcão, C. \& Széchy, M.T.M. 2005. Changes in shallow phytobenthic assemblages in southeastern Brazil, following the replacement of Sargassum vulgare (Phaeophyta) by Caulerpa scalpelliformis (Chlorophyta). Botanica Marina 48: 208-217.

Guiry, M.D. \& Guiry, G.M. [on line]. Algaebase. Word-wide eletronic publication, National University of Ireland, Galway. Disponível na Internet via www.url: http://www.algaebase.org. (Acesso em: 4/abril/2007).

Littler, M.M. \& Littler, D.S. 1984. Relationships between macroalgal functional form groups and substrata stability in a subtropical rocky-intertidal system. Journal of Experimental Marine Biology and Ecology 74: 13-34.

Reis, R.P. \& Yoneshigue-Valentin, Y. 1998. Variação espaço-temporal de populações de Hypnea musciformis (Rhodophyta, Gigartinales) na baía de Sepetiba e Armação dos Búzios, Rio de Janeiro, Brasil. Acta Botanica Brasilica 13: 465-83

Reis, R.P.; Caldeira, A.Q.; Miranda, A.P.S.M. \& Barros-Barreto, M.B. 2006. Potencial para maricultura da carragenófita Hypnea musciformis (Wulfen) J.V. Lamour. (Gigartinales - Rhodophyta) na ilha da Marambaia, Rio de Janeiro, Brasil. Acta Botanica Brasilica 20: 763-769.

Reis, R.P.; Bastos, M. \& Góes, H.G. 2007. Cultivo de Kappaphycus alvarezii no litoral do Rio de Janeiro. Panorama da Aqüicultura 17: 42-47.

SEMADS - Secretaria do Estado de Meio Ambiente e Desenvolvimento Sustentável do Estado do Rio de Janeiro. 2001.
Bacias Hidrográficas e Recursos Hídricos da Macroregião Ambiental 2 - Bacia da baía de Sepetiba. Rio de Janeiro.

Silveira, M.P. 2004. Aplicação do biomonitoramento para avaliação da qualidade da água em rios. Empresa Brasileira de Pesquisa Agropecuária. Centro Nacional de Pesquisa de Monitoramento e Avaliação de Impacto Ambiental. Ministério da Agricultura, Pecuária e Abastecimento. Embrapa Meio Ambiente, Documentos 36. Jaguariúna, SP.

Silva, M.A. \& Araújo, F.G. 2003. Influência das variáveis ambientais na fauna acompanhante na pesca da manjuba Anchoa tricolor Agassiz (Actinopterygii, Engraulidae) na baía de Sepetiba, Rio de Janeiro. Revista Brasileira de Zoologia 20: $367-371$.

Secretaria do Estado de Meio Ambiente e Desenvolvimento Sustentável do Estado do Rio de Janeiro. 2001. Bacias Hidrográficas e Recursos Hídricos da Macroregião Ambiental 2 - Bacia da baía de Sepetiba. Rio de Janeiro.

Steneck, R.S. \& Dethier, M.N. 1994. A functional group approach to the structure of algal-dominated communities. Oikos 69: 476-498.

Széchy, M.T.M. \& Paula, E.J. 1997. Macroalgas epífitas em Sargassum (Phaeophyta - Fucales) do litoral dos estados do Rio de Janeiro e São Paulo, Brasil. Leandra 12: 1-10.

Széchy, M.T.M. \& Paula, E.J. 2000. Padrões estruturais quantitativos de bancos de Sargassum (Phaeophyta - Fucales) do litoral dos estados do Rio de Janeiro e São Paulo, Brasil. Revista Brasileira de Botânica 23: 121-132.

Széchy, M.T.M.; Amado Filho, G.M.; Cassano, V.; Paula, J.C.; BarrosBarreto, M.B.; Reis, R.P.; Marins-Rosa, B.V. \& Moreira, F.M. 2005. Levantamento florístico das macroalgas da baía de Sepetiba e adjacências, RJ: ponto de partida para o Programa GloBallast no Brasil. Acta Botanica Brasilica 19: 587-596.

Wynne, M.J. 2005. A checklist of benthic marine algae of the tropical and subtropical western Atlantic: first revision. Nova Hedwigia 129: $1-152$.

Zar, J.H. 1996. Biostatistical analysis. New Jersey, PrenticeHall International Editions. 